\title{
Existencia y substancia del mundo en el Tractatus*
}

\author{
Javier Vidal
}

Universidad de Concepción

Department of Philosophy

C/ Beltrán Mathieu 15 A Barrio Universitario, Concepcion

Chile

fravidal@udec.cl

Article info

CDD: 160

Received: 19.07.2017; Accepted: 13.11.2017

DOI: http://dx.doi.org/10.1590/0100-6045.2018.V41N1.JV

\section{Keywords:}

Espacio lógico

Logical space

Última línea

Bottom line

Existencia necesaria

Necessary existence

Pasaje sobre la substancia

Substance passage

Objetos tractarianos

Independencia

Tractarian objects

Independence

\section{RESUMEN}

En este artículo trato de establecer que, según el Tractatus, la posibilidad de pensar que el espacio lógico fuera vacío es equivalente a la posibilidad de pensar que el mundo no hubiera existido. Tal representación viene dada por la última línea de una tabla de verdad que es una descripción completa del espacio lógico: es la línea en la que todas las proposiciones elementales son falsas. En primer lugar, argumentaré que una proposición elemental sería falsa -más que no tener un valor de verdad- si fuera evaluada con respecto a un mundo posible en el que alguno de los objetos nombrados en esa proposición no existe. A continuación, tendré que mostrar que es posible que los objetos tractarianos no hubieran existido para poder concluir que es posible pensar que los objetos tractarianos no hubieran existido. Por último, estoy obligado a ofrecer una nueva interpretación del pasaje sobre la substancia (T 2.021-2.0212).

* En relación a algunas dudas sobre el original alemán del Tractatus, agradezco la ayuda que me prestaron los profesores Martin Fricke, de la Universidad Nacional Autónoma de México, y Ezequiel Zerbudis, de la Universidad Nacional del Litoral, Argentina. Agradezco también las observaciones de los árbitros de esta revista.

Manuscrito - Rev. Int. Fil. Campinas, v. 41, n. 1, pp. 33-66, jan.-mar. 2018. 


\begin{abstract}
In this paper I intend to establish that, according to the Tractatus, the possibility of thinking that the logical space were empty is equivalent to the possibility of thinking that the world had not existed. Such a representation is given by the bottom line of a truth-table which is a complete description of logical space: it is the line where all elementary propositions are false. Firstly, I will argue that an elementary proposition would be false -rather than lacking a truth-value- if it were evaluated with respect to a possible world in which some named object in that proposition does not exist. Then I will have to show that it is possible that the tractarian objects had not existed in order to conclude that it is possible to think that the tractarian objects had not existed. Finally, I am bound to offer a new interpretation of the substance passage (T 2.021-2.0212).
\end{abstract}

En este artículo trato de establecer que en el Tractatus la posibilidad de imaginarse o pensar que el espacio lógico fuera vacío ( $\mathrm{T} 2.013)^{1}$ equivale a la posibilidad de pensar que el mundo no hubiera existido (o, equivalentemente, que los objetos tractarianos no hubieran existido). La última línea de una tabla de verdad que es una descripción completa del espacio lógico corresponde a esta representación de la inexistencia del mundo: se trata de la línea en la que todas las proposiciones ${ }^{2}$ elementales son falsas. En defensa de la posibilidad de pensar que el mundo no hubiera existido formularé un argumento según el cual una proposición elemental sería falsa -más que no tener ningún valor de verdad- si fuera evaluada con respecto a un mundo posible en el que no existe alguno de los objetos nombrados en esa proposición. Pero, en ese caso, establecer que, mediante esa última línea, es posible pensar que los objetos no hubieran existido (es decir, que es posible pensar que el mundo no hubiera existido) depende aún de establecer que es posible que los objetos no hubieran existido (es decir, que es posible que el mundo no hubiera existido). Esta es, además, una aplicación del isomorfismo, o identidad de forma, entre el lenguaje y el mundo según el cual lo que es representable/pensable y lo que es posible se corresponden por completo. Ahora bien, el hilo argumental empleado con anterioridad también llevará a la conclusión de que, en efecto, los objetos

\footnotetext{
${ }^{1}$ Sin dejar de tener en cuenta las traducciones clásicas al inglés del Tractatus, me guiaré principalmente por la traducción al español de Valdés Villanueva (2003). Ocasionalmente me desviaré de esta traducción. Para las referencias a los aforismos de la obra seguiré el sistema que acabo de emplear en el texto.
}

2 Mantendré el uso tractariano de "proposición” [Satz] para hablar de las oraciones.

Manuscrito - Rev. Int. Fil. Campinas, v. 41, n. 1, pp. 33-66, jan.-mar. 2018. 
tractarianos no existen necesariamente. Por ello, tendré que ofrecer, además, una explicación o interpretación del pasaje sobre la substancia del mundo ( $T$ 2.021-2.0212) de la cual no se siga que los objetos existen necesariamente.

\section{I}

Wittgenstein sostiene que, dadas todas las proposiciones elementales verdaderas, tenemos una descripción completa del mundo (T 4.26). Pues, el mundo es la totalidad de los estados de cosas existentes (T 2.04) ${ }^{3}$. También se dice que la totalidad de los pensamientos verdaderos es una figura del mundo (T 3.01) ${ }^{4}$. En este sentido, el mundo es todo lo que es el caso (T 1). Ahora bien, si el objetivo es especificar el sentido de una proposición, se necesita, más que una descripción completa del mundo, una descripción completa del espacio lógico ${ }^{5}$, ya que el sentido de una proposición determina un lugar en el espacio lógico ( $\mathrm{T}$ 3.4) mediante una división de ese espacio entre los mundos posibles con los que esa proposición está de acuerdo, de modo que es verdadera en tales mundos, y los mundos posibles con los que esa proposición no está de acuerdo, de modo que es falsa en tales mundos. En otras palabras, el lugar que ocupa una proposición en el espacio lógico es el conjunto de mundos posibles en los

3 Estaré ateniéndome a la versión, que recoge la traducción al español de Valdés Villanueva, según la cual un hecho [Tatsache], o lo que es el caso, es la existencia de estados de cosas (T 2). Podría decirse que la existencia de un solo estado de cosas es un hecho atómico. A su vez un estado de cosas [Sachverbalt] es una combinación de objetos (T 2.01). Por último, aunque a veces parece no distinguirse de un hecho, una situación [Sacblage] comprende posibles estados de cosas. Pues, Wittgenstein suele hablar de situaciones posibles ( $\mathrm{T} 2.202,3.11,4.125,4.462,5.525)$. Su caso límite es un solo estado de cosas (cf. Cerezo 2005, pp. 277-285).

${ }^{4}$ Un pensamiento es una proposición con sentido ( $\mathrm{T}$ 4).

${ }^{5}$ Desde la teoría figurativa de la proposición, el espacio lógico puede ser concebido en términos de las posibilidades de existencia y no existencia de los estados de cosas. Desde la teoría veritativo-funcional de la proposición, el espacio lógico puede ser concebido en términos de las posibilidades de verdad de las proposiciones elementales. Pero, no hay ningún conflicto aquí, dado que las posibilidades de verdad de las proposiciones elementales significan las posibilidades de existencia y no existencia de los estados de cosas (T 4.3).

Manuscrito - Rev. Int. Fil. Campinas, v. 41, n. 1, pp. 33-66, jan.-mar. 2018. 
que esa proposición es verdadera (Cerezo 2012, p. 649). En efecto, esta es una lectura en términos de mundos posibles de la idea de que la realidad queda fijada por una proposición de modo que sólo caben dos alternativas: sí o no ( $\mathrm{T}$ 4.023). Por tanto, debe tenerse en cuenta la distinción entre el estatus de una proposición dentro de un mundo posible y el estatus de esa proposición con respecto a un mundo posible (Carruthers 1990, pp. 121-122) ${ }^{6}$. Una proposición con sentido dentro del mundo real puede ser evaluada con respecto a mundos posibles distintos del mundo real dentro de los cuales esa proposición no es un hecho ( $\mathrm{O}$, aunque es un hecho, no adquiere un sentido por falta de una coordinación de sus nombres constituyentes con objetos en esos mundos). Es decir, una proposición con sentido dentro del mundo real puede ser evaluada con respecto a mundos posibles dentro de los cuales no hay lenguaje ni pensamiento. Más aún, estamos diciendo que para que una proposición adquiera un sentido dentro del mundo real tiene que poder evaluarse con respecto a todos los mundos posibles, incluyendo aquellos dentro de los cuales no hay lenguaje ni pensamiento.

Supongamos entonces que tenemos la siguiente tabla de verdad:

$$
\begin{aligned}
& \begin{array}{lllllll}
\mathrm{p}_{1} & \mathrm{p}_{2} & \mathrm{p}_{3} & \mathrm{p}_{4} & \ldots & \ldots & \mathrm{p}_{n}
\end{array}
\end{aligned}
$$

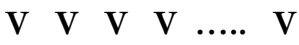

$$
\begin{aligned}
& \text { V } \quad \mathrm{V} \quad \mathrm{V} \quad \mathrm{V} \quad \ldots . . \mathrm{F}
\end{aligned}
$$

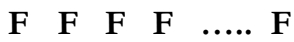

Supongamos ahora que la primera línea de esta tabla es una descripción completa del mundo, en la medida en que corresponde a la verdad de las proposiciones elementales que son verdaderas en el mundo real. Podría pensarse entonces que esa tabla de verdad es una descripción completa del

\footnotetext{
${ }^{6}$ En términos contemporáneos esta distinción corresponde, em parte, a la distinción de Kaplan entre contexto de emisión y circunstancia de evaluación (Kaplan 1989; Proops 2004, pp. 114-116).
}

Manuscrito - Rev. Int. Fil. Campinas, v. 41, n. 1, pp. 33-66, jan.-mar. 2018. 
espacio lógico. Sin embargo, esta conclusión no es correcta, pues esa tabla no incluye las proposiciones elementales que son falsas cuando son evaluadas con respecto al mundo real ni considera, por tanto, todos los mundos posibles con respecto a los cuales puede ser evaluada una proposición. De hecho, la última línea de la tabla no puede representar la posibilidad de que ningún estado de cosas hubiera existido, dado que no excluye que alguna de las proposiciones elementales que son falsas en el mundo real sea verdadera en un mundo posible en el que son falsas todas las proposiciones elementales que son verdaderas en el mundo real. En otras palabras, la última línea de esa tabla no es la línea consistente en que todas las proposiciones elementales son falsas. Esto significa que una tabla de verdad que es una descripción completa del espacio lógico debe ser completada con todas las proposiciones elementales que son falsas en el mundo real. Ahora bien, la realidad es precisamente la existencia y no existencia de estados de cosas ( $\mathrm{T}$ 2.06). Pero,Wittgenstein dice también en la segunda parte de T 4.26 que el mundo queda descrito completamente una vez que se dan todas las proposiciones elementales con la indicación adicional de cuáles de ellas son verdaderas y cuáles de ellas son falsas. Por tanto, describir el mundo de esta manera es describir la realidad (T 2.063). Desde luego, una vez que están dadas todas las proposiciones elementales verdaderas queda determinado qué proposiciones elementales son falsas: la totalidad de los estados de cosas existentes determina también qué estados de cosas no existen ( $T$ 2.05). Consideremos entonces la siguiente tabla de verdad como una descripción completa del espacio lógico cuya línea intermedia es una descripción completa del mundo/de la realidad:

Manuscrito - Rev. Int. Fil. Campinas, v. 41, n. 1, pp. 33-66, jan.-mar. 2018. 


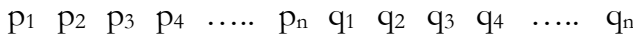

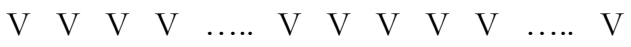

$$
\begin{aligned}
& \begin{array}{lllllllllllll}
\mathbf{V} & \mathbf{V} & \mathbf{V} & \mathbf{V} & \ldots & \ldots & \mathrm{V} & \mathrm{F} & \mathrm{F} & \mathrm{F} & \mathrm{F} & \ldots \ldots & \mathrm{F}
\end{array}
\end{aligned}
$$

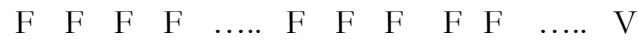

$$
\begin{aligned}
& \begin{array}{lllllllllllll}
F & F & F & F & \ldots & F & F & F & F & F & \ldots \ldots & F
\end{array}
\end{aligned}
$$

Entonces, la penúltima línea de esta tabla representa un mundo posible en el que todas las proposiciones elementales que son verdaderas en el mundo real son falsas en ese mundo, pero al menos otra proposición elemental es verdadera en ese mundo. En cambio, la última línea de la tabla es la línea consistente en que todas las proposiciones elementales son falsas. Por tanto, la última línea de esa tabla representa la posibilidad de que ningún estado de cosas hubiera existido 7 . Ahora bien, hay una forma de sostener que, a pesar de eso, la última línea no representa la posibilidad de que el mundo no hubiera existido (Maury 1977, pp. 150-153; Page 1997). Supongamos que los objetos que constituyen los estados de cosas pudiesen existir aisladamente, es decir, pudiesen existir sin combinarse con otros objetos en algún estado de cosas. Esta suposición está motivada por la tesis de que, como se lee frecuentemente el pasaje del Tractatus sobre la substancia ( $T$ 2.021-2.0212), los objetos

\footnotetext{
${ }^{7}$ Equivalentemente, esa posibilidad es representada por una proposición, digamos " $\varphi$ ", que es la conjunción de las negaciones de todas las proposiciones elementales. En efecto, " $\varphi$ " es una proposición que sólo es verdadera cuando todas las proposiciones elementales son falsas (Cerezo 2012, p. 651; 2005, pp. 262-264, 275-277).
}

Manuscrito - Rev. Int. Fil. Campinas, v. 41, n. 1, pp. 33-66, jan.-mar. 2018. 
tractarianos existen necesariamente ${ }^{8}$. Pues, si los objetos no pudiesen existir aisladamente, la posibilidad de que ningún estado de cosas hubiera existido sería equivalente a la posibilidad de que los objetos no hubieran existido. Pero, dado que los objetos existen necesariamente, la última línea de la tabla representaría precisamente la posibilidad de que los objetos hubieran existido sin combinarse con otros objetos en estados de cosas. Así, el mundo posible en el que todas las proposiciones elementales son falsas sería un mundo en el que los objetos existen aisladamente. Como es claro, en ese caso la última línea de la tabla no representaría la posibilidad de que el mundo no hubiera existido.

Sin embargo, esta interpretación depende crucialmente de asumir una lectura extravagante del Tractatus según la cual es posible que los objetos existan aisladamente. Una parte crucial de mi argumentación en la siguiente sección depende de una lectura ortodoxa según la cual los objetos tractarianos no pueden existir aisladamente. Pero es una lectura tan bien motivada ${ }^{9}$ que si la única interpretación de la última línea de una tabla de verdad que es una descripción completa del espacio lógico implicase la posibilidad de que los objetos hubieran existido aisladamente, debería concluirse que esa última línea es un absurdo en términos tractarianos (Reinhardt 2005, p. 342). Téngase en cuenta aquí, en particular, el aforismo donde Wittgenstein dice que puede imaginarse un espacio lógico vacío pero no un objeto sin espacio (T 2.013). Aunque justamente está en cuestión qué significa imaginarse un espacio lógico vacío, si es o no imaginarse un espacio lógico sin objetos (es decir, la posibilidad de que los objetos no hubieran existido), es claro que imaginarse un objeto sin espacio lógico sería imaginarse un objeto sin combinarse con otros objetos en estados de cosas. Pues, desde un punto de vista ontológico el

\footnotetext{
${ }^{8}$ Aparte de Proops (2004), que examinaré en la próxima sección, otra reciente defensa de esta lectura se encuentra en Tejedor (2003). Por el contrario, recientemente Frascolla ha negado esta lectura sobre la base de que los objetos tractarianos son entidades abstractas universales, específicamente qualia, de las cuales no tiene sentido predicar la existencia en primer lugar (Frascolla 2004, pp. 375-376). Así, sólo los complejos/estados de cosas son entidades concretas del mundo. Por eso, la posibilidad de que ningún estado de cosas hubiera existido es directamente la posibilidad de que el mundo no hubiera existido (Frascolla 2004, pp. 372-374).

9 Cuando introduzca la tesis tractariana de que los objetos no pueden existir aisladamente comentaré algunos de los aforismos que motivan decisivamente esta lectura.
}

Manuscrito - Rev. Int. Fil. Campinas, v. 41, n. 1, pp. 33-66, jan.-mar. 2018. 
espacio lógico en el que un objeto está es precisamente un espacio de posibles estados de cosas, como se empieza estableciendo en T 2.013. Pero en ese aforismo se concluye precisamente que no es posible imaginarse un objeto sin espacio.

Entre los intérpretes clásicos, quizá Carruthers es quien ofrece un examen más exhaustivo de las distintas alternativas a la hora de reconstruir el pasaje sobre la substancia como un argumento cuya conclusión es la existencia necesaria de objetos simples (Carruthers 1990, pp. 85-106). En este punto quiero detenerme solamente en evitar un malentendido que, como señala Carruthers, atraviesa algunas de estas alternativas. Ciertamente, en un mundo posible en el que no existe alguno de los objetos nombrados en una proposición elemental, esa proposición no puede adquirir un sentido (ni, por tanto, una condición de verdad). En efecto, según el Tractatus la coordinación de los elementos de una figura con las cosas, como un modo de llegar hasta la realidad, es una condición necesaria para que esa figura pueda representar una situación (T 2.151-2.1515). Por tanto, la coordinación de nombres con objetos es una condición necesaria para que una proposición elemental adquiera un sentido. Pero de ahí no es legítimo concluir que los objetos tractarianos existen en todos los mundos posibles. Una cosa es que los objetos nombrados en una proposición elemental deban existir en los mundos posibles dentro de los cuales esa proposición adquiere un sentido. Otra cosa es que los objetos nombrados en una proposición elemental deban existir en los mundos posibles con respecto a los cuales esa proposición puede ser evaluada como verdadera o falsa. El requisito de que los objetos nombrados existan es un requisito acerca de los mundos posibles dentro de los cuales una proposición elemental adquiere un sentido en primer lugar, no acerca de los mundos posibles con respecto a los cuales esa proposición puede ser evaluada después ${ }^{10}$. Así, una proposición elemental que adquiere un sentido dentro del mundo real mediante

${ }^{10} \mathrm{~A}$ este respecto, no puede negarse que imaginar un espacio lógico vacío es imaginar un espacio lógico sin objetos sobre la base de que si no hubiera objetos, entonces no habría espacio lógico (Gale 1976, p. 95). Sin duda, para imaginar un espacio lógico es necesario que dentro del mundo en el que tiene lugar ese imaginar existan los objetos que hacen posible ese imaginar. De ahí no se sigue que no cabe imaginar un espacio lógico que contenga la posibilidad de que ningún objeto hubiera existido. Consideremos la siguiente analogía. Para pensar cualquier cosa, es necesario que yo exista. De ahí no se sigue, obviamente, que no puedo pensar la posibilidad de que yo no hubiera existido.

Manuscrito - Rev. Int. Fil. Campinas, v. 41, n. 1, pp. 33-66, jan.-mar. 2018. 
una coordinación de nombres con objetos en ese mundo puede ser evaluada después con respecto a todos los mundos posibles, incluyendo aquellos mundos dentro de los cuales esa proposición no adquiere un sentido por falta de una coordinación de nombres con objetos en esos mundos. En principio, una proposición elemental que adquiere un sentido dentro del mundo real porque sus nombres constituyentes tienen referentes en ese mundo puede ser evaluada después con respecto a un mundo posible en el que tales referentes no existen (Carruthers 1990, pp. 93, 99, 101, 121-122) ${ }^{11}$.

Mi objetivo en este artículo es una aproximación al problema motivada por la interpretación de Proops (2004). Supongamos que una proposición elemental no tendría un valor de verdad -más que ser falsa- si fuera evaluada con respecto a un mundo posible en el que alguno de los objetos nombrados en esa proposición no existe. Entonces, recurriendo a una generalización, resultaría que no es posible pensar que el mundo no hubiera existido mediante la última línea de una tabla de verdad que es una descripción completa del espacio lógico. En efecto, ninguna proposición elemental tendría un valor de verdad -más que ser falsa- cuando es evaluada con respecto a la posibilidad de que los objetos no hubieran existido. Que todas las proposiciones elementales sean falsas no podría representar la posibilidad de que los objetos no hubieran existido. Pero teniendo en cuenta que el sentido de una proposición consiste en una división del espacio lógico tal que para todo mundo posible sólo caben dos alternativas: sí o no, es decir, que la proposición sea verdadera o sea falsa en ese mundo, podría concluirse de ahí que, puesto que algunas proposiciones tienen sentido, los objetos deben existir necesariamente. Si no, ninguna proposición tendría sentido dado que no tendría un valor de verdad en los mundos posibles en los que alguno de los objetos nombrados en esa proposición no existe. A

\footnotetext{
11 Por su parte, Carruthers propone una reconstrucción del argumento en términos de la prioridad, para la comprensión lingüística, de las proposiciones singulares en relación a las proposiciones generales. Si los objetos tractarianos existiesen contingentemente, entonces la comprensión de una proposición singular/elemental requeriría conocer la implicación de una generalización existencial: el hablante debería saber que esa proposición singular sólo es verdadera en un mundo posible si en ese mundo existen objetos que son idénticos a los objetos nombrados en la proposición. Pero conocer esta implicación es contrario a la idea de que la comprensión de una proposición singular no requiere la comprensión previa de una proposición general (Carruthers 1990, pp. 117126).
}

Manuscrito - Rev. Int. Fil. Campinas, v. 41, n. 1, pp. 33-66, jan.-mar. 2018. 
continuación voy a argumentar, primero, que una proposición elemental sería falsa -más que no tener un valor de verdad- si fuera evaluada con respecto a un mundo posible en el que no existe alguno de los objetos nombrados en esa proposición. En ese caso, la última línea de la tabla podría representar la posibilidad de que el mundo no hubiera existido. Pero, como es obvio, que esa línea pueda ser interpretada de tal manera aún va a depender de establecer la posibilidad misma de que el mundo no hubiera existido (es decir, la posibilidad misma de que los objetos no hubieran existido). La posibilidad de que los objetos no hubieran existido es, obviamente, una presuposición de interpretar esa última línea como la línea en la que todas las proposiciones elementales son falsas -más que no tener un valor de verdad- cuando son evaluadas con respecto a esa posibilidad.

\section{II}

Consideremos ahora el pasaje en el que Wittgenstein afirma que los objetos son la substancia del mundo:

2.021 Los objetos forman la substancia del mundo. Por ello no pueden ser compuestos.

2.0211 Si el mundo careciese de substancia, el que una proposición tuviera sentido dependería de que otra proposición fuese verdadera.

2.0212 Sería entonces imposible trazar una figura del mundo (verdadera o falsa).

Proops (2004) sostiene una interpretación según la cual este pasaje constituye un argumento a favor de la existencia necesaria de objetos simples. Presentaré ese argumento como si T 2.0211 y T 2.0212 fuesen las premisas cuya conclusión es T 2.021'2. Supongamos primero que una proposición con el

12 Esta interpretación del pasaje es más explícita en Zalabardo (2012, pp. 126-132), quien, no obstante, considera que es equivocada. Otra reconstrucción del argumento está en Morris (2008, pp. 358-361), quien lo introduce como el argumento de la plenitud

Manuscrito - Rev. Int. Fil. Campinas, v. 41, n. 1, pp. 33-66, jan.-mar. 2018. 
nombre "a" tiene sentido13. Este es el argumento reconstruido en sus pasos esenciales para ese caso particular:

(A) Si el objeto a no existiera en todo mundo posible, entonces el que una proposición con el nombre "a" tuviera sentido con respecto a un mundo posible dependería de que otra proposición acerca de la existencia de a fuese verdadera cuando es evaluada con respecto a ese mundo ( $\mathrm{T}$ 2.0211).

Pues, supongamos que en este contexto tener o no tener sentido con respecto a un mundo posible es equivalente a tener o no tener un valor de verdad en ese mundo (Proops 2004, p. 116). Supongamos, además, que una proposición con el nombre "a" no tendría un valor de verdad -más que ser falsa- cuando es evaluada con respecto a un mundo posible en el que el objeto $a$ no existe. Entonces, dado que una proposición con el nombre "a" no tendría un valor de verdad en un mundo posible en el que el objeto $a$ no existe, se sigue que si a no existiera en todo mundo posible, esa proposición no tendría un valor de verdad en un mundo posible ( $\mathrm{y}$ así no tendría sentido con respecto a ese mundo) a menos que fuese verdadera en ese mundo una proposición que establece la existencia de $a$ (Proops 2004, pp. 116-117). El punto relevante es que, a pesar de optar por esta interpretación, Proops reconoce que es una cuestión disputable si una proposición con el nombre "a" no tendría un valor

del valor de verdad, pero también da una interpretación alternativa. El argumento contiene otras premisas que, sin embargo, sólo incluiré en los comentarios a (A), (B) y (C), para preservar la importancia de la interpretación del pasaje sobre la substancia como núcleo argumental. En el mismo sentido, aunque la totalidad de los aforismos sobre la substancia son, más bien, los comprendidos ente T 2.02 y T 2.0272, sigo a Zalabardo en destacar solamente los que corresponden al núcleo argumental.

${ }_{13}$ Mientras no indique lo contrario, una proposición con el nombre "a" o bien es una proposición elemental o bien es una proposición compuesta de alguna proposición elemental con el nombre "a". Como es sabido, los nombres genuinos son en primer lugar los constituyentes de las proposiciones elementales en las que termina el análisis lógico ( $T$ 4.22). Sin embargo, de que un objeto sea nombrado no se sigue que ese objeto sea simple más que compuesto hasta que precisamente se establezca la conclusión del argumento que estamos examinando. Cuando sea preciso, indicaré explícitamente que una proposición con el nombre "a" es una proposición elemental.

Manuscrito - Rev. Int. Fil. Campinas, v. 41, n. 1, pp. 33-66, jan.-mar. 2018. 
de verdad o, por el contrario, sería falsa en un mundo posible en el que $a$ no existe (Proops 2004, p. 121) ${ }^{14}$. En cualquier caso, consideremos ahora el paso siguiente:

(B) Entonces, una proposición con el nombre "a" no podría tener sentido (T 2.0212).

Propiamente hablando, el establecimiento de (B) requiere volver a la tesis de que una proposición con el nombre " $a$ " no tendría un valor de verdad en un mundo posible en el que el objeto $a$ no existe. Es decir, más que (A) debemos tener en cuenta la siguiente tesis:

$(\mathrm{A}+)$ Si el objeto a no existiera en algún mundo posible, entonces una proposición con el nombre "a" no tendría un valor de verdad - más que ser falsa- cuando es evaluada con respecto a ese mundo (y así no tendría sentido con respecto a ese mundo).

Ahora bien, como ya vimos en la primera sección, parece ser una tesis tractariana que una proposición no tiene sentido en absoluto si no puede ser evaluada como verdadera o falsa con respecto a todo mundo posible. Digamos que esta es la tesis tractariana del sentido como plenitud del valor de verdad. No se trata sólo de que si a no existiera en algún mundo posible entonces una proposición con el nombre "a" no tendría sentido con respecto a ese mundo. En principio, esta consecuencia podría armonizarse con la idea de que esa proposición sigue teniendo sentido con respecto a los mundos posibles en los que $a$ existe, en la medida en que tiene un valor de verdad en tales mundos (es decir, es verdadera o falsa en tales mundos). Pero esta armonización es

\footnotetext{
${ }^{14}$ Hay un conflicto de la opción de Proops con la afirmación de Wittgenstein de que una proposición acerca de un complejo será falsa -más que un sinsentido- en el caso de que ese complejo no exista (T 3.24). Pero Proops sostiene que T 3.24 ya asume que hay una substancia del mundo por debajo de los complejos (2004, p. 117). Otro problema para la opción de Proops es dar cuenta de las proposiciones existenciales negativas. Pero Proops muestra que las proposiciones existenciales negativas también plantean un problema importante a la opción según la cual una proposición es falsa -más que no tener un valor de verdad- en un mundo posible en el que no existe alguno de los objetos nombrados en esa proposición (2004, p. 125, nota 41).
}

Manuscrito - Rev. Int. Fil. Campinas, v. 41, n. 1, pp. 33-66, jan.-mar. 2018. 
contraria a la tesis tractariana de que, como podría expresarse de forma más contemporánea, una proposición con sentido divide completamente el espacio lógico entre los mundos posibles con los que acuerda, de modo que es verdadera en tales mundos, y los mundos posibles con los que no acuerda, de modo que es falsa en tales mundos. En efecto, la proposición tiene que describir la realidad de manera completa, lo que hace cuando la realidad queda fijada por la proposición de modo que sólo caben dos alternativas: sí o no ( $\mathrm{T}$ 4.023, 5.156; Proops 2004, pp. 117-120). De este modo, una proposición con el nombre "a" que no tuviera un valor de verdad en algún mundo posible no podría tener sentido en absoluto ${ }^{15}$.

Pero habíamos empezado por suponer que una proposición con el nombre "a" tiene sentido, contrariamente a (B). Por tanto,

(C) El objeto a existe en todo mundo posible. Por ello no puede ser compuesto (T 2.021) (por A+ y B).

En efecto, los complejos son agregados de cosas que pueden dejar de existir, dado que cualquier estado de cosas o situación sólo existe contingentemente, como veremos más adelante ${ }^{16}$.

Es claro que de las premisas de este argumento se sigue que, aunque fuera posible que el mundo no hubiera existido, no sería posible pensar que el mundo no hubiera existido. Supongamos que, como el objeto $a$, ninguno de los objetos nombrados en las proposiciones elementales existe en todo mundo posible. Entonces, dado que para cada una de las proposiciones elementales habría al menos un mundo posible en el que esa proposición no tiene un valor de verdad (por $\mathrm{A}+$ ), se sigue de la tesis tractariana del sentido como plenitud del valor de verdad que ninguna proposición elemental tendría sentido en absoluto (por B). Es decir, no podríamos tener una descripción completa del espacio lógico ni,

15 Una objeción inmediata a esta interpretación del pasaje relevante es que (B) no es implicado por (A) más la tesis tractariana del sentido como plenitud del valor de verdad, sino que sólo es implicado por $(\mathrm{A}+)$ más esa tesis tractariana.

${ }_{16}$ Una vez que se concluye que un objeto genuinamente nombrado, como $a$, es un objeto simple, puede describirse el caso más básico de complejo como $a \mathrm{R} b$. No me detendré más en la cuestión de la simplicidad de los objetos, que no es el tema de este artículo.

Manuscrito - Rev. Int. Fil. Campinas, v. 41, n. 1, pp. 33-66, jan.-mar. 2018. 
por tanto, una representación de la posibilidad de que el mundo no hubiera existido (entre las otras posibilidades en el espacio lógico que tampoco serían representables). Esta es precisamente la conclusión general establecida en T 2.0212: sería imposible trazar una figura del mundo (verdadera o falsa).

Mi objetivo ahora es argumentar que $(\mathrm{A}+)$ no es correcto sino que, por el contrario, se sigue de otras tesis tractarianas que si un objeto a no existe en algún mundo posible, entonces una proposición elemental con el nombre "a" es falsa -más que no tener un valor de verdad- en ese mundo ${ }^{17}$. Pero entonces la premisa $(\mathrm{A}+)$ no puede usarse para probar que el objeto a tiene una existencia necesaria, lo que significa que debe darse otra interpretación del pasaje sobre la substancia del mundo (como haré en la sección III). Además, en esta segunda sección se concluirá, primero, que si una proposición elemental con el nombre "a" es falsa cuando es evaluada con respecto a un mundo posible en el que el objeto $a$ no existe, entonces sería posible pensar que el mundo no hubiera existido mediante la última línea de una tabla de verdad que es una descripción completa del espacio lógico. Con todo, establecer que es posible pensar que el mundo no hubiera existido mediante la última línea de esa tabla aún dependerá de establecer que es posible que el mundo no hubiera existido (es decir, que es posible que los objetos no hubieran existido). Pues, sin la posibilidad de que los objetos no hubieran existido, no tendría sentido evaluar las proposiciones elementales con respecto a esa posibilidad (ni, por tanto, evaluarlas como falsas con respecto a esa posibilidad). Ahora bien, siguiendo el mismo hilo argumental empleado contra la premisa $(\mathrm{A}+)$ se llegará a la conclusión fundamental de que los objetos tractarianos no existen necesariamente.

En la presentación del argumento estaré considerando, por simplicidad de la argumentación, que sólo hay dos proposiciones elementales con el nombre "a" (pues, nada importante depende del número $n$ de proposiciones elementales, como espero que resultará evidente):

${ }^{17} \mathrm{El}$ argumento que Proops lee en el pasaje sobre la substancia, y con ello la premisa $(\mathrm{A}+)$, es acerca de cualquier proposición con el nombre "a". Por tanto, si una proposición elemental con el nombre "a" es falsa en un mundo posible en el que $a$ no existe, se sigue que la premisa $(\mathrm{A}+)$ no es correcta.

Manuscrito - Rev. Int. Fil. Campinas, v. 41, n. 1, pp. 33-66, jan.-mar. 2018. 
(1) Las proposiciones elementales con el nombre "a" son "Fa" y "Ga".

Como es obvio, estas proposiciones representan estados de cosas en los que un objeto a podría ocurrir. Estaré asumiendo también que si sólo hay dos proposiciones elementales con el nombre "a", entonces sólo hay dos estados de cosas en los que el objeto a podría ocurrir. Esta es una consecuencia del isomorfismo, o identidad de forma, entre el lenguaje y el mundo según el cual las posibilidades de combinación de los nombres en proposiciones elementales se corresponden por completo con las posibilidades de combinación de los objetos nombrados en estados de cosas. Pues, la noción de forma es la noción de una posibilidad de combinación. Esto es explícito cuando se dice que la forma de un objeto es la posibilidad de su ocurrencia en estados de cosas ( $T$ 2.0141). También cuando se dice que la forma de figuración es la posibilidad de que los objetos se relacionen unos con otros como lo hacen los elementos de la figura (T 2.151). Por tanto, si la forma lógica es lo que las figuras lógicas, así como específicamente las proposiciones como figuras de la realidad ( $\mathrm{T}$ 4.01), deben tener en común con el mundo para poder representarlo ( $\mathrm{T}$ 2.16-2.171), se sigue que el lenguaje (o el pensamiento) y el mundo comprenden las mismas posibilidades de combinación. Esta es, por tanto, la manera tractariana de expresar la idea de que lo que es representable/pensable y lo que es posible se corresponden por completo ${ }^{18}$.

${ }^{18}$ Cuando Wittgenstein se expresa de esta última manera sólo establece directamente que lo que es pensable es posible, pero no lo converso ( $T$ 3.02). Sin embargo, ello se debe a que aquí le interesa destacar que no se puede pensar algo ilógico (T 3.03). Pero también se sigue de otros aforismos que todo lo que es posible es pensable, como cuando dice que las proposiciones pueden representar toda la realidad ( $T$ 4.12). Esto parece ser una consecuencia inmediata de la tesis de que la forma lógica es la forma de la realidad (T 2.18, 4.121). Como también lo señala en las lecciones de 1930-1932, pp. 9-10. Incluso si se afirmase que según el Tractatus son posibles otros espacios lógicos con otros objetos cuyas combinaciones en estados de cosas no serían pensables por nosotros (Cerezo 2012, p. 652; 2005, pp. 264, 272-274), eso no afectaría a la tesis de que, dados ciertos objetos en la realidad que nosotros podemos representarnos, como el objeto $a$, cualquier posible estado de cosas que tenga a $a$ como constituyente es pensable por nosotros.

Manuscrito - Rev. Int. Fil. Campinas, v. 41, n. 1, pp. 33-66, jan.-mar. 2018. 
A continuación introduciré la tesis tractariana básica sobre la conexión esencial entre objetos y estados de cosas que ya vimos en la primera sección:

(2) Los objetos son dependientes de estados de cosas en el sentido de que no pueden existir aisladamente (T 2.0122).

En la segunda parte de T 2.0122 Wittgenstein se refiere a esta tesis mediante una analogía con las palabras: es imposible que las palabras aparezcan tanto aisladas como en la proposición. Se trata de invocar el principio del contexto según el cual sólo en la trabazón de una proposición tiene significado un nombre ( $\mathrm{T}$ 3.3 ${ }^{19}$. Más importante que esta analogía, ya se estableció previamente en el Tractatus que es esencial a un objeto que pueda ser parte constituyente de un estado de cosas (T 2.011). Pues, si un objeto puede ocurrir en un estado de cosas, esa posibilidad debe estar ya prejuzgada en el objeto ( $T$ 2.012). Pero si un objeto pudiese existir aisladamente, entonces sería accidental que encajase posteriormente en una situación (T 2.0121). Veamos el argumento que Wittgenstein plantea en este pasaje ( $\mathrm{T}$ 2.011-2.0124) de este otro modo. Si un objeto pudiese existir aisladamente de manera que fuera ontológicamente autosuficiente, entonces la posibilidad de que ocurriera en un estado de cosas no sería una posibilidad necesaria: aunque es posible que ocurriera en ese estado de cosas, podría no haberlo sido. Así como no es necesario que un objeto ocurra en un determinado estado de cosas, tampoco sería necesario que ese objeto pudiera ocurrir en ese estado. Nada en la naturaleza del objeto determinaría que esa posibilidad de ocurrencia fuese esencial a él. Sin embargo, lo que es posible es necesariamente posible ${ }^{20}$ : si algo puede ser verdadero de un objeto le es esencial a ese objeto que eso pueda ser verdadero de él. Por tanto, los objetos no pueden existir aisladamente (Morris 2008, pp. 33-35) ${ }^{21}$.

19 Esta misma referencia al principio del contexto para explicar la existencia de los objetos como constituyentes de estados de cosas se encuentra en las lecciones de 19301932, p. 119.

${ }^{20}$ Algo lógico no puede ser meramente posible (T 2.0121).

21 Téngase en cuenta, sin embargo, que Morris no plantea directamente esta interpretación como un argumento contra la existencia aislada o independiente de los objetos.

Manuscrito - Rev. Int. Fil. Campinas, v. 41, n. 1, pp. 33-66, jan.-mar. 2018. 
Recordemos ahora que de la interpretación de Proops, en particular de $(\mathrm{A}+)$, se sigue:

(3) La proposición elemental "Fa" no tiene un valor de verdad más que ser falsa- cuando es evaluada con respecto a un mundo posible en el que el objeto $a$ no existe.

Entonces, se sigue:

(4) La proposición " $\sim \mathrm{Fa} \rightarrow \mathrm{Ga}$ " es una tautología -es verdadera en todo mundo posible en el que "Fa" tiene un valor de verdad(por 1, 2 y 3).

En efecto, según (3) cualquier mundo posible en el que la proposición "Fa" tiene un valor de verdad es un mundo en el que el objeto $a$ existe. Por tanto, cualquier mundo posible en el que "Fa" es falsa será un mundo en el que $a$ existe. Ese será un mundo en el que no existe el estado de cosas Fa. Pero dado que sólo hay dos proposiciones elementales con el nombre "a", se sigue que la proposición "Ga" es verdadera en ese mundo. Pues, si el objeto $a$ existe en ese mundo, entonces tiene que ocurrir en el estado de cosas $G a$. En consecuencia, la proposición " $\sim \mathrm{Fa} \rightarrow \mathrm{Ga"}$ es una tautología (por 1, 2 y 3). Si "Fa" es verdadera en un mundo posible, entonces se sigue trivialmente que " $\sim \mathrm{Fa} \rightarrow$ $\mathrm{Ga}$ " es verdadera en ese mundo. $\mathrm{Si}$ "Fa" es falsa en un mundo posible, entonces se sigue que " $\sim \mathrm{Fa} \rightarrow \mathrm{Ga}$ " es verdadera en ese mundo (dado que, según el análisis anterior, "Ga" tiene que ser verdadera en ese mundo) 22 . Veámoslo de este otro modo. Ex hypothesi la proposición " $\sim \mathrm{Fa} \rightarrow \mathrm{Ga}$ " sólo sería falsa en un mundo posible en el que "Fa" es falsa y "Ga" es falsa. Pero,

22 Esto implica también, obviamente, que las proposiciones elementales no serían lógicamente independientes entre sí, contrariamente a lo que es una tesis tractariana fundamental ( $T$ 5.152, 5.134). Sin embargo, téngase en cuenta que, para algunos autores, parte de la importancia del pasaje sobre la substancia está relacionada con una posible incoherencia interna entre la tesis de la independencia lógica de las proposiciones elementales y la teoría figurativa de la proposición, lo que a veces se traduce en un abandono de esa tesis como esencial al Tractatus (Ginet 1973; Gale 1976; Wedin 1992). 
dado que sólo hay dos proposiciones elementales con el nombre "a", ese sería un mundo posible en el que el objeto $a$ no existe. Pues, no habrá ningún estado de cosas en el que $a$ ocurre. Entonces, según (3) ese será un mundo posible en el que "Fa" no tiene un valor de verdad -más que ser falsa- (así como "Ga" tampoco tiene un valor de verdad en ese mundo). Contrariamente a la hipótesis inicial, ese será un mundo posible en el que " $\sim \mathrm{Fa} \rightarrow \mathrm{Ga}$ " no tiene un valor de verdad -más que ser falsa-. Por tanto, se sigue efectivamente que la proposición " $\sim \mathrm{Fa} \rightarrow \mathrm{Ga}$ " es verdadera en todo mundo posible en el que la proposición "Fa" tiene un valor de verdad (por 1, 2 y 3) ${ }^{23}$. Téngase en cuenta que si la proposición "Fa" fuera falsa -más que no tener un valor de verdad- cuando es evaluada con respecto a un mundo posible en el que el objeto a no existe, la proposición " $\sim \mathrm{Fa} \rightarrow \mathrm{Ga"}$ no sería una tautología. Dado que el objeto $a$ no existe en ese mundo, no habrá ningún estado de cosas en el que $a$ ocurre, de modo que tanto "Fa" como "Ga" serán falsas en ese mundo. Entonces, la proposición " $\sim \mathrm{Fa} \rightarrow \mathrm{Ga}$ ” también será falsa en ese mundo.

Ahora bien, es claro que la proposición " $\sim \mathrm{Fa} \rightarrow \mathrm{Ga}$ ” no es una tautología. En contradicción con (4), esto llevaría a la negación de (3) ${ }^{24}$. Pero podría

23 Es claro que el número de proposiciones elementales con el nombre "a" no es relevante aquí. Supongamos que hay un número cualquiera $n$ de proposiciones elementales con el nombre "a”. Entonces, en vez de (1) tendríamos, por ejemplo:

(1*) Las proposiciones elementales con el nombre "a” son "Fa”, "Ga”, "Ha”, "Ia".... " $\Phi^{\mathrm{n}} \mathrm{a}$ ".

En vez de (4) tendríamos ahora:

$\left(4^{*}\right)$ La proposición “ $\sim \mathrm{Fa} \rightarrow\left(\mathrm{Ga} \vee \mathrm{Ha} \vee \mathrm{Ia} \ldots . . \Phi^{\mathrm{n}} \mathrm{a}\right)$ ” es una tautología -es verdadera en todo mundo posible en el que "Fa" tiene un valor de verdad-.

Pues bien, podría argumentarse a partir de (1*), (2) y (3), como en el texto principal, para ver fácilmente la tautologicidad que debería tener esa proposición (sin embargo, no lo haré aquî). El problema es que, al igual que veremos en el caso de " $\sim \mathrm{Fa} \rightarrow \mathrm{Ga}$ ", esa proposición tampoco es tautológica en términos de estructura veritativo-funcional, lo que genera una contradicción.

24 Pues, por el favor del argumento no está en cuestión la verdad de (1) ni de (2). En particular, (2) parece ser una tesis tractariana mucho más segura, como he tratado de

Manuscrito - Rev. Int. Fil. Campinas, v. 41, n. 1, pp. 33-66, jan.-mar. 2018. 
objetarse que la proposición " $\sim \mathrm{Fa} \rightarrow \mathrm{Ga}$ " no es una tautología precisamente si la proposición "Fa" es falsa -más que no tener un valor de verdad- en un mundo posible en el que el objeto a no existe, como acabo de mostrar. La objeción que podría hacérseme ahora es que estaría considerando que la proposición " $\sim \mathrm{Fa} \rightarrow \mathrm{Ga}$ " no es una tautología, que es correcto si la proposición "Fa" es falsa en un mundo posible en el que el objeto a no existe, para concluir a continuación que la proposición "Fa" es falsa en un mundo posible en el que el objeto $a$ no existe! Sin embargo, no es esta mi línea de argumentación. En el Tractatus la afirmación de que una proposición es o no es implicada por otra no depende de ninguna consideración que vaya más allá de la estructura:

(5) Que la verdad de una proposición se sigue de la verdad de otras proposiciones es algo que puede verse a partir de la estructura de las proposiciones ( $\mathrm{T}$ 5.13).

Como es sabido, esta es una doctrina medular del Tractatus: las relaciones lógicas entre proposiciones, más que estar determinadas extrínsecamente por principios lógicos o reglas de inferencia (como en la lógica de Frege y Russell), deben ser intrínsecas/internas a las proposiciones que están en esas relaciones (T 5.131-5.132, 5.2, 6.12; Proops 2000, pp. 89-93; Ricketts 1996, pp. 63-64, 8283). Pues, las proposiciones de la lógica ponen de manifiesto las relaciones lógicas entre proposiciones mediante tautologías que no dicen nada ( $\mathrm{T}$ 6.11, 6.121). Por ello, podríamos habérnoslas sin tales tautologías: en una notación idónea podemos reconocer directamente las relaciones lógicas entre proposiciones mediante la mera inspección de su estructura (T 6.113, 6.122). Más aún, puede argumentarse que la teoría veritativo-funcional de la proposición está motivada por esta epistemología tractariana de la lógica según la cual debemos ser capaces de ver las relaciones lógicas inspeccionando directamente la estructura de las proposiciones. La estructura veritativofuncional de las proposiciones contiene la información que se requiere para determinar si una proposición se sigue o no de otra porque especifica, para cada proposición, de qué modo los valores de verdad de esa proposición dependen

mostrar, que (3), que, por otro lado, está en conflicto con T 3.24, como también vimos (cf. nota 14).

Manuscrito - Rev. Int. Fil. Campinas, v. 41, n. 1, pp. 33-66, jan.-mar. 2018. 
de los valores de verdad de las proposiciones elementales. En este sentido, las tablas de verdad son una notación que permite la inspección directa de esta estructura veritativo-funcional. Mediante las tablas de verdad podemos entonces ver si una proposición se sigue de otra examinando simplemente si los fundamentos veritativos de la primera (es decir, los valores de verdad de las proposiciones elementales que hacen verdadera esa proposición) están contenidos en los fundamentos veritativos de la segunda (T 5.101-5.121). Pero, dado que la estructura veritativo-funcional contiene la información que se requiere para determinar si una proposición se sigue o no de otra, tenemos que no es necesaria ninguna información adicional acerca de los estados de cosas de cuya existencia o no existencia dependen los valores de verdad de esas proposiciones (Zalabardo 2015, pp. 191-194)25. Por tanto,

(6) La proposición “ $\sim \mathrm{Fa} \rightarrow \mathrm{Ga}$ " no es una tautología (por 5).

Pues, si ex hyphotesi esta proposición fuera una tautología eso no se vería a partir de la estructura veritativo-funcional de las proposiciones " $\sim \mathrm{Fa}$ " y "Ga". En términos de estructura veritativo-funcional una proposición no se sigue de la otra: son independientes entre sí dado que, de entrada, no tienen ningún argumento veritativo (es decir, ninguna proposición elemental) en común ( $\mathrm{T}$ $5.152)^{26}$. No se trata de objetar la tautologicidad de " $\sim \mathrm{Fa} \rightarrow \mathrm{Ga}$ " considerando lo que ocurriría si la proposición "Fa" fuese falsa en un mundo posible en el que $a$ no existe, como se cuestionaba. Pues, esto significaría tener ya en cuenta una consideración acerca de la estructura ontológica del mundo posible con respecto al cual está siendo evaluada la proposición "Fa" (a saber: que es un mundo en el que $a$ no existe), algo que va más allá de la estructura veritativofuncional de "Fa" y que por ello es información adicional que no se requiere,

${ }^{25}$ Sin duda que este es uno de los sentidos en los que puede decirse que la lógica es a priori (T 5.133). A este respecto, Zalabardo habla de formalismo epistémico (2015, p. 191).

26 Esta es la razón por la que estoy formulando el argumento en términos de proposiciones elementales. Si "Fa" y "Ga" no fueran proposiciones elementales, entonces la estructura veritativo-funcional de esas proposiciones como funciones de verdad de proposiciones elementales podría mostrar que la proposición " $\sim \mathrm{Fa} \rightarrow \mathrm{Ga}$ " es una tautología. Por ejemplo, si al término del análisis en proposiciones elementales obtuviéramos la proposición " $\sim(p \wedge \sim q) \rightarrow(p \vee q)$ ". 
según Wittgenstein, para determinar si una proposición se sigue o no de otra. Del mismo modo, que la proposición " $\sim \mathrm{Fa} \rightarrow \mathrm{Ga}$ " fuera una tautología sería una consecuencia, contrariamente a (5), de que la proposición "Fa" no tiene un valor de verdad en un mundo posible en el que el objeto a no existe ${ }^{27}$. En efecto, según (5) esa proposición no es una tautología. El problema entonces es que obtenemos una contradicción:

(7) La proposición “ $\sim \mathrm{Fa} \rightarrow \mathrm{Ga}$ ” es y no es una tautología (por 4 y 6).

Teniendo en cuenta que no está en cuestión la verdad de (1) ni de (2), se sigue finalmente:

(8) La proposición elemental "Fa" es falsa -más que no tener un valor de verdad- cuando es evaluada con respecto a un mundo posible en el que el objeto $a$ no existe (por 3 y 7 ).

Liberándonos ahora de la restricción ad hoc a dos proposiciones elementales con el nombre " $a$ ", diremos que todas las proposiciones elementales con el nombre "a" son falsas en un mundo posible en el que el objeto a no existe. Pero la misma conclusión es válida para cualquier conjunto de proposiciones elementales que tienen un cierto nombre común como constituyente. Por tanto, sería posible pensar que el mundo no hubiera existido mediante la última línea de una tabla de verdad que es una descripción completa del espacio lógico, interpretada o leída como una línea donde todas las proposiciones elementales son falsas cuando son evaluadas con respecto a la posibilidad de que los objetos no hubieran existido. Con todo, la justificación de esta interpretación depende aún de establecer la posibilidad misma de que los objetos no hubieran existido. Como es obvio, la posibilidad de que los objetos no hubieran existido es una presuposición de interpretar esa última línea como la línea en la que todas las

27 Desde luego, tanto en un caso como en otro se hace uso también de otra información adicional que no está contenida en la estructura veritativo-funcional de las proposiciones: el conocimiento del número de proposiciones elementales con el nombre "a", así como el principio ontológico de que los objetos no pueden existir aisladamente.

Manuscrito - Rev. Int. Fil. Campinas, v. 41, n. 1, pp. 33-66, jan.-mar. 2018. 
proposiciones elementales son falsas cuando son evaluadas con respecto a esa posibilidad. Pues bien, siguiendo el mismo hilo argumental que llevó hasta (8) estableceré ahora que los objetos tractarianos no existen necesariamente. Empecemos con la tesis contraria para un caso particular:

(9) El objeto $a$ existe en todo mundo posible.

Entonces, al introducir nuevamente las premisas (1) y (2), se sigue:

(10) La proposición “ $\sim \mathrm{Fa} \rightarrow \mathrm{Ga}$ " es una tautología -es verdadera en todo mundo posible en el que "Fa" tiene un valor de verdad(por 1, 2 y 9).

En efecto, es una consecuencia trivial de (9) que cualquier mundo posible en el que la proposición " $\mathrm{Fa}$ " es falsa será un mundo en el que el objeto $a$ existe. Ese será un mundo en el que no existe el estado de cosas Fa. Pero dado que sólo hay dos proposiciones elementales con el nombre "a", se sigue que la proposición "Ga" es verdadera en ese mundo. Pues, si el objeto $a$ existe en ese mundo, entonces tiene que ocurrir en el estado de cosas $G a$. En consecuencia, la proposición " $\sim \mathrm{Fa} \rightarrow \mathrm{Ga}$ " es una tautología (por 1, 2 y 9). Si "Fa" es verdadera en un mundo posible, entonces se sigue trivialmente que " $\sim \mathrm{Fa} \rightarrow$ $\mathrm{Ga}$ " es verdadera en ese mundo. $\mathrm{Si}$ "Fa" es falsa en un mundo posible, entonces se sigue que " $\sim \mathrm{Fa} \rightarrow \mathrm{Ga}$ " es verdadera en ese mundo (dado que, según el análisis anterior, "Ga" tiene que ser verdadera en ese mundo). Veámoslo así. Ex hypothesi la proposición " $\sim \mathrm{Fa} \rightarrow \mathrm{Ga}$ " sólo sería falsa en un mundo posible en el que "Fa" es falsa y "Ga" es falsa. Pero, dado que sólo hay dos proposiciones elementales con el nombre "a", ese sería un mundo posible en el que el objeto $a$ no existe. Pues, no habrá ningún estado de cosas en el que $a$ ocurre. Entonces, según (9) ese mundo no es posible. Contrariamente a la hipótesis inicial, no hay ningún mundo posible en el que la proposición " $\sim \mathrm{Fa}$ $\rightarrow \mathrm{Ga}$ " sea falsa. Por tanto, se sigue efectivamente que la proposición " $\sim \mathrm{Fa} \rightarrow$ Ga" es una tautología (por 1, 2 y 9$)^{28}$.

${ }_{28}$ Nuevamente este resultado no cambia en función del número $n$ de proposiciones elementales con el nombre "a".

Manuscrito - Rev. Int. Fil. Campinas, v. 41, n. 1, pp. 33-66, jan.-mar. 2018. 
Otra vez, esa proposición no sería una tautología en términos de estructura veritativo-funcional sino, entre otras cosas, en términos de la información adicional de que el objeto $a$ existe en todo mundo posible ${ }^{29}$. En efecto, según (5) esa proposición no es una tautología. Por tanto, se obtiene nuevamente una contradicción:

(11) La proposición “ $\sim \mathrm{Fa} \rightarrow \mathrm{Ga}$ " es y no es una tautología (por 6 y 10).

Teniendo en cuenta otra vez que no está en cuestión la verdad de (1) ni de (2), se sigue finalmente:

(12) El objeto $a$ no existe en todo mundo posible (por 9 y 11).

Generalizando esta conclusión se sigue que ningún objeto tractariano existe necesariamente. Para cada objeto es posible construir tautologías absurdas en términos de estructura veritativo-funcional si ese objeto existiera en todo mundo posible. Entonces, dado que es una consecuencia de (8) que todas las proposiciones elementales son falsas -más que no tener un valor de verdadcuando son evaluadas con respecto a la posibilidad de que los objetos no hubieran existido, que resulta ser una posibilidad genuina, se sigue que la última línea de una tabla de verdad que es una descripción completa del espacio lógico puede ser interpretada como una representación de la posibilidad de que los objetos no hubieran existido (es decir, de la posibilidad de que el mundo no hubiera existido) ${ }^{30}$.

${ }^{29}$ Incluso si aceptáramos que la existencia necesaria de los objetos es un requisito de la teoría figurativa de la proposición, no parece ser un requisito de la teoría veritativofuncional de la proposición (es decir, de la proposición como función de verdad de proposiciones elementales). Pero la explicación de las tautologías en términos de estructura viene dada por la teoría veritativo-funcional.

30 Téngase en cuenta otra vez que si las proposiciones elementales no tuviesen un valor de verdad -más que ser falsas- cuando son evaluadas con respecto a un mundo posible en el que no existe alguno de los objetos nombrados en ellas, no podríamos tener una representación de la posibilidad de que los objetos no hubieran existido. En realidad, por la tesis tractariana del sentido como plenitud del valor de verdad no podríamos tener ninguna representación en absoluto. Entonces, aunque fuera posible que los

Manuscrito - Rev. Int. Fil. Campinas, v. 41, n. 1, pp. 33-66, jan.-mar. 2018. 


\section{III}

Es obligado ofrecer ahora una explicación o interpretación del pasaje sobre la substancia del mundo (T 2.021-2.0212), diferente de la interpretación según la cual ahí se argumenta acerca de la existencia necesaria de objetos simples, como hace Proops.

Wittgenstein sostiene que un objeto es independiente en el sentido de que puede ocurrir en toda situación posible ( $\mathrm{T}$ 2.0122). Evidentemente se trata de todas las situaciones posibles que residen en la naturaleza del objeto ( $T$ 2.0123). Quiero proponer que este es el sentido en el que los objetos tractarianos son la substancia del mundo (T 2.021). De hecho, se dice que la substancia es lo que subsiste independientemente de lo que es el caso ( $T$ 2.024). Pero es claro que se está hablando del mismo sentido de independencia: un objeto es independiente en el sentido de que puede ocurrir en cualquier situación posible si y sólo si subsiste independientemente de lo que es el caso, en el sentido de que subsiste independientemente de las situaciones en las que de hecho ocurre. Esta forma de independencia de los objetos no significa, sin embargo, que los objetos puedan existir aisladamente, teniendo en cuenta que en la segunda parte de $\mathrm{T}$ 2.0122 se establece, como ya vimos, que tal independencia es a la vez una forma de conexión con estados de cosas y, por tanto, una forma de dependencia ${ }^{31}$. Se trata de que si un objeto ocurre de hecho en una determinada situación (es decir, si esa situación existe), entonces es posible que no hubiera ocurrido en esa situación (es decir, es posible que esa situación no hubiera existido). En otras palabras, se trata de que, para un objeto dado, hay al menos un mundo posible en el que ese objeto no ocurre en una situación determinada. Por tanto, toda situación o estado de cosas es contingente. En este punto se pone el énfasis en que un objeto subsiste independientemente de lo que es el caso. Pero puede decirse equivalentemente que si un objeto no ocurre de hecho en una

objetos no hubieran existido, como acabamos de mostrar, no sería posible pensar que los objetos no hubieran existido. Pero este resultado va contra la tesis tractariana según la cual lo que es representable/pensable y lo que es posible se corresponden por completo.

31 Para evitar confusiones podría decirse que los objetos son independientes ${ }_{1}$ (en el sentido de poder ocurrir en toda situación) pero no son independientes 2 (en el sentido de poder existir aisladamente). Pero creo que es suficiente el contexto para entender cuál es el sentido de independencia que se está aplicando. 
determinada situación (es decir, si esa situación no existe), entonces es posible que hubiera ocurrido en esa situación (es decir, es posible que esa situación hubiera existido). Resulta que, para un objeto dado, hay al menos un mundo posible en el que ese objeto ocurre en una situación determinada. Aquí se pone el énfasis en que un objeto es independiente en el sentido de que puede ocurrir en cualquier situación posible ${ }^{32}$. Ciertamente, de este sentido de independencia se sigue que, frente a los cambiantes e inestables estados de cosas, los objetos son la forma permanente que un mundo posible tiene en común con el mundo real (T 2.022-2.023, 2.026-2.0271).

Sin embargo, esto no implica que los objetos tractarianos existen necesariamente. Considérese más bien que la independencia de lo que es el caso y la existencia necesaria de los objetos están en conflicto (en conjunción con otras tesis tractarianas). En efecto, en la teoría veritativo-funcional de la proposición es fundamental el pensamiento de que de la existencia o no existencia de un estado de cosas no se sigue la existencia o no existencia de otro estado de cosas (T 2.061-2.062). Esta es una consecuencia de que las proposiciones elementales son lógicamente independientes entre sí ( $T$ 5.152, 5.134). En particular, de la no existencia de un estado de cosas en un mundo posible no se sigue la existencia de algún otro estado de cosas en ese mundo. Por tanto, si cualquier estado de cosas es contingente en el sentido de que es posible que no hubiera existido, entonces es posible que ningún estado de cosas hubiera existido. Pero, dado que los objetos no pueden existir aisladamente, eso significa que es posible que los objetos no hubieran existido. Parece así que, contrariamente a la independencia de lo que es el caso, la existencia necesaria de un objeto requiere que haya al menos un determinado estado de cosas que existe en todo mundo posible. De este modo, si un objeto ocurre de hecho en ese estado de cosas, entonces ese objeto ocurre en ese estado de cosas en todo mundo posible. Ese objeto existiría necesariamente porque ese estado de cosas existe necesariamente.

Ofreceré ahora una interpretación según la cual el pasaje sobre la substancia presenta un argumento a favor de la existencia independiente de objetos

32 En efecto, supongamos que tenemos el siguiente principio de contingencia: $\mathrm{A} \rightarrow \diamond$ $\sim$ A. Entonces, del mismo modo que de "p" se obtiene " $\diamond \sim p$ " por ese principio, también de " $\sim \mathrm{p}$ " se sigue " $\vee \mathrm{p}$ " por ese principio (más la ley de eliminación de la doble negación).

Manuscrito - Rev. Int. Fil. Campinas, v. 41, n. 1, pp. 33-66, jan.-mar. 2018. 
simples. Consideraré nuevamente un caso particular. Supongamos de entrada que una proposición con el nombre "a" tiene sentido. Para ese caso particular tenemos:

(A*) Si el objeto $a$ no fuera independiente en tanto que no pudiera ocurrir en alguna situación, entonces el que una proposición con el nombre "a" tuviera sentido dependería de que otra proposición acerca de la posibilidad de la situación articulada en esa proposición fuese verdadera (T 2.0211).

Si el objeto a no fuera independiente, eso significaría que hay al menos una situación con $a$ como constituyente que no puede existir. Pues, evidentemente, no se trata aquí de las situaciones que existen o pueden existir pero de las que $a$ no sería un constituyente. Se trata de que el objeto $a$ no puede ocurrir en una situación porque esa situación con a como constituyente no puede existir. Es decir, ex hyphotesi estamos ante una situación o estado de cosas con a como constituyente que es imposible. Pero supongamos que intentamos hablar o pensar acerca de una situación imposible mediante una proposición con el nombre "a". Ciertamente Wittgenstein sostiene que una proposición es una combinación gramatical de nombres que por ello mismo ya contiene la posibilidad de su sentido. Aunque no contiene un sentido, ya está articulada para poder expresarlo (T 3.13-3.142) ${ }^{33}$. Pero su sentido consiste en el acuerdo y desacuerdo con las posibilidades de existencia y no existencia de estados de cosas

33 Considero adecuadas aquellas interpretaciones del Tractatus, que luego pueden diferir puntualmente (Morris 2008, pp. 158-169; Johnston 2007), según las cuales una proposición, como una combinación gramatical o legítima de nombres, es un símbolo al que aún falta darle un sentido (o significado). Se reconoce el símbolo en el signo atendiendo a su uso 'significativo' [sinnvollen], que es el uso de acuerdo a las leyes de la sintaxis lógica (T 3.326; Morris 2008, pp. 164-166; Johnston 2007, pp. 383-384). Pero en la sintaxis lógica no está permitido que el significado de un signo desempeñe papel alguno (T 3.33). Por eso también se dice que la proposición contiene la forma, pero no el contenido, de su sentido ( $T$ 3.13). Por su sintaxis lógica como símbolo una proposición ya contiene la posibilidad de su sentido: está articulada (T 3.251) como la situación posible que podría representar. Para que tenga sentido aún es necesario darle significado a los nombres que están combinados gramaticalmente en esa proposición ( $T$ $5.473,5.4733)$.

Manuscrito - Rev. Int. Fil. Campinas, v. 41, n. 1, pp. 33-66, jan.-mar. 2018. 
(T 4.2). Entonces, una proposición ya contiene la posibilidad de la situación que representa ( $T$ 3.02). Así, una proposición no puede representar una situación imposible (T 3.03). Por tanto, parece que si intentáramos pensar acerca de una situación imposible no podríamos estar usando una proposición consistente en una combinación gramatical de nombres sino, más bien, una mera ristra de marcas o sonidos sin articulación alguna. Pero esta conclusión está también determinada porque Wittgenstein tampoco encuentra coherente la idea de una situación o estado de cosas que es imposible: en particular, un estado de cosas es una combinación de objetos cuya posibilidad está determinada por la naturaleza de tales objetos ( $\mathrm{T}$ 2.01-2.0141). No tiene sentido la idea de una combinación imposible de objetos. Se trataría, por así decirlo, de que hubiese una combinación tal que los objetos no pudiesen estar combinados de esa manera. De este modo, no podemos usar una proposición en el intento de pensar acerca de una situación imposible precisamente porque no hay nada sobre lo que pensar que esté articulado como la proposición lo está.

Sin embargo, en este punto de la argumentación tenemos que asumir la posición de quien encuentra coherente la idea de una situación imposible. Entonces, nos encontraríamos en el siguiente escenario. Por un lado, el intento de pensar acerca de una situación imposible como algo que está articulado, al igual que una situación posible, nos comprometerá con una proposición como una combinación gramatical de nombres ${ }^{34}$. Por otro lado, en la medida en que esa situación es imposible no consideraremos que esa proposición contiene la posibilidad de su sentido, dado que una proposición no puede representar una situación imposible. Digamos que, en ese caso, una cierta proposición con el nombre "a" será una pseudo-representación de una situación imposible. Pero,

\footnotetext{
34 Podría optarse también por decir que, para quien encuentra coherente la idea de una situación imposible, el intento de representar esa situación viene dado por una combinación ilegítima (o agramatical) de nombres. Como una combinación de nombres, se trataría aún de una proposición más que de una mera ristra de marcas o sonidos, a diferencia de lo que se sostiene en el Tractatus, según el cual solo caben proposiciones como combinaciones legítimas de nombres (Morris 2008, pp. 167-168). Encuentro difícil conjugar esta propuesta con la idea de que, a pesar de todo, esa proposición estaría articulada. En cualquier caso, no me parece relevante decidir entre una u otra opción en la medida en que en ambos casos tendríamos que una proposición con el nombre "a" puede representar una situación posible o pseudo-representar una situación imposible.
}

Manuscrito - Rev. Int. Fil. Campinas, v. 41, n. 1, pp. 33-66, jan.-mar. 2018. 
en este contexto dialéctico, resulta que una proposición con el nombre "a" que, por el contrario, llegue a representar una situación posible tampoco contendrá la posibilidad de su sentido. Pues, según acabamos de ver, el hecho de que una proposición sea una combinación gramatical de nombres no implicará que pueda tener sentido (ni, por tanto, que llegue a representar más que pseudorepresentar). Entonces, dado que mediante una proposición con el nombre "a" estaríamos representándonos una situación posible o, por el contrario, estaríamos pseudo-representándonos una situación imposible, se sigue que el sentido de una proposición con el nombre "a" dependerá de que otra proposición acerca de la posibilidad de la situación articulada en esa proposición sea verdadera. Si ex bypothesi hay al menos una situación imposible con el objeto a como constituyente que podemos pseudo-representarnos mediante una proposición, entonces el hecho de que una proposición con el nombre "a" tenga sentido (es decir, represente una situación posible) va a depender de una garantía externa acerca de la posibilidad de la situación articulada en esa proposición. Supongamos ahora que la proposición con el nombre "a" es la proposición elemental "Fa". Tenemos que el sentido de esta proposición va a depender de que la proposición "Es posible que $\mathrm{Fa}$ " sea verdadera. Si esta última proposición es verdadera, entonces "Fa" es una proposición con sentido mediante la cual nos representamos un posible estado de cosas. Si esa proposición es falsa, entonces "Fa" es un sinsentido mediante el cual nos pseudo-representamos un estado de cosas que es imposible ${ }^{35}$. Consideremos ahora el paso siguiente:

\footnotetext{
35 Existen al menos dos interpretaciones alternativas del aforismo T 2.0211 que también interpretan el consecuente "el que una proposición tuviera sentido dependería de que otra proposición fuese verdadera" como en $\left(A^{*}\right)$. En el caso de Morris se argumenta que una proposición como "Es posible que Fa" no es contingentemente verdadera, que es como tendría que ser si los objetos no constituyesen la forma permanente del mundo. Pero precisamente el antecedente de T 2.0211 es que los objetos no serían la forma permanente del mundo (Morris 2008, pp. 42-47). En el caso de Zalabardo se argumenta que una proposición como "Es posible que Fa" (que diría, equivalentemente, que el objeto $a$ es combinable con otros objetos de una cierta manera) no es una proposición genuina según el Tractatus porque no hay hechos 'formales', es decir, no hay hechos acerca de las propiedades combinatorias de los objetos. Esto es una consecuencia de que tales propiedades son internas a los objetos. Pero precisamente el antecedente de T 2.0211 es que las propiedades combinatorias de los objetos no son internas (Zalabardo 2015, pp. 142-146, 166-173, 181-184).
}

Manuscrito - Rev. Int. Fil. Campinas, v. 41, n. 1, pp. 33-66, jan.-mar. 2018. 
(B) Entonces, una proposición con el nombre "a" no podría tener sentido (T 2.0212).

Desde luego, es claro que, según Wittgenstein, el hecho de que "Fa" sea una combinación gramatical de nombres que puede representar un posible estado de cosas no puede resultar determinado desde fuera de esa proposición. La combinación gramatical de nombres en una proposición ya puede representar una combinación de objetos en un posible estado de cosas. No es que una combinación gramatical de nombres en una proposición requiera la garantía externa de que haya objetos que pueden combinarse de la misma manera para que esa proposición pueda representar un posible estado de cosas. Esta es precisamente la tesis de que una proposición ya contiene la posibilidad de su sentido antes de dar significado a sus nombres constituyentes mediante la coordinación con objetos. Por eso, una vez que se ha dado significado a los nombres, se dice que la proposición muestra su sentido, es decir, muestra cómo están las cosas si es verdadera (T 4.022). Cómo están combinados los nombres a los que se ha dado significado en la proposición ya muestra cómo pueden estar combinados los objetos nombrados en un estado de cosas. En consecuencia, el sentido de la proposición " $\mathrm{Fa}$ " no puede depender de que la proposición "Es posible que Fa" sea verdadera, como establece el consecuente de $\left(A^{*}\right)$. Sin embargo, esta argumentación no puede aplicarse aquí porque, en el contexto dialéctico de $\left(\mathrm{A}^{*}\right)$, es directamente una petición de principio.

La razón por la que la proposición "Fa" no podría tener sentido es que, dado el consecuente de $\left(\mathrm{A}^{*}\right)$, el sentido de la proposición "Es posible que Fa" no sería independiente de $s u$ propia verdad, lo que es contrario a la independencia entre sentido y verdad que es constitutiva de la teoría tractariana del sentido ${ }^{36}$. Una parte importante de este argumento es que el sentido de la

\footnotetext{
${ }^{36}$ En vez de considerar esta razón alguien podría argumentar, de manera diferente, que el problema es que una proposición como "Es posible que $\mathrm{Fa}$ " no puede tener sentido (T 5.525, 4.124). Parece que es así porque esa proposición sería un intento de decir o representar lo que la proposición "Fa" y el estado de cosas $\mathrm{Fa}$ tienen en común, a saber: una posibilidad de combinación. Precisamente la noción de forma lógica es la noción de una posibilidad de combinación. Pero, como acabamos de señalar, "Fa" ya muestra esta posibilidad de combinación. Entonces, puesto que lo que puede mostrarse no puede decirse ( $T$ 4.1212), se sigue que "Es posible que Fa" no puede tener sentido.
}

Manuscrito - Rev. Int. Fil. Campinas, v. 41, n. 1, pp. 33-66, jan.-mar. 2018. 
proposición "Es posible que $\mathrm{Fa}$ " requiere que, como una parte integral de ella, la proposición "Fa" ya tenga sentido. De entrada, si "Fa" no tuviera sentido es porque, a pesar de ser una combinación gramatical de nombres, no habríamos dado significado a alguno de esos nombres ( $T$ 5.473, 5.4733, 6.53). Pero en el contexto dialéctico de $\left(\mathrm{A}^{*}\right)$ diríamos más bien que si "Fa" no tuviera sentido es porque, a pesar de ser una combinación gramatical de nombres, es el intento de pensar o hablar acerca de un estado de cosas que es imposible ${ }^{37}$. Entonces, puesto que "Fa" es una parte integral de "Es posible que Fa", esta proposición tampoco tendría sentido. En efecto, una proposición es un sinsentido cuando otra proposición que es una parte integral de ella es un sinsentido ${ }^{38}$. Veámoslo de manera inversa. Como dice Wittgenstein en el contexto de cuestionar la teoría de Russell acerca del juicio como relación múltiple: la explicación correcta de la forma de la proposición "A juzga que p" debe mostrar que es imposible que "p" sea un sinsentido como parte integral de esa proposición ( $\mathrm{T}$ 5.5422; Notes on Logic, pp. 96-97). Del mismo modo, la explicación correcta de la forma de la proposición "Es posible que Fa", es decir, la explicación según la cual esa proposición puede tener sentido ${ }^{39}$, debe mostrar que es imposible que

Evidentemente, se requiere aquí una explicación independiente de lo que hace que no pueda decirse o representarse lo que también puede mostrarse. En cualquier caso, esta respuesta no es viable aquí, pues en el contexto dialéctico de $\left(\mathrm{A}^{*}\right)$ vuelve a ser una petición de principio.

${ }^{37}$ En principio, estas explicaciones del sinsentido no son excluyentes. Podría decirse que si una proposición, que es una combinación gramatical de nombres, no puede representar una situación imposible es porque no se le ha dado significado a alguno de esos nombres. Pero, supongamos que en el caso de una proposición con el nombre "a" el problema es que no se le ha dado significado a ese nombre. Entonces, resulta paradójicamente que mediante esa proposición no se podría siquiera pseudorepresentar una situación imposible que tuviera como constituyente el objeto $a$.

${ }^{38}$ En el Tractatus, así como la proposición "Fa" no tendría sentido porque una parte integral de ella, un nombre, no habría recibido un significado, la proposición "Es posible que Fa" no tendría sentido porque una parte integral de ella, precisamente "Fa", no tendría sentido por esa razón.

39 Desde luego, parece que según el Tractatus "Es posible que Fa" no puede tener sentido (cf. nota 36). En cualquier caso, el punto sigue siendo válido: una explicación según la cual esa proposición pudiera tener sentido debería mostrar que es imposible que "Fa" no tenga sentido como parte integral de esa proposición.

Manuscrito - Rev. Int. Fil. Campinas, v. 41, n. 1, pp. 33-66, jan.-mar. 2018. 
"Fa" sea un sinsentido como parte integral de esa proposición. En otras palabras, esa explicación debe mostrar que, como una parte integral de "Es posible que Fa", necesariamente "Fa" tiene sentido. Supongamos entonces que, como establece el consecuente de $\left(\mathrm{A}^{*}\right)$, el sentido de la proposición "Fa" depende de la verdad de la proposición "Es posible que Fa". De aquí se sigue que, de acuerdo a una explicación según la cual la proposición "Es posible que Fa" puede tener sentido, el sentido de "Es posible que Fa" no será independiente de su propia verdad. Pero este resultado es contrario a la independencia entre sentido y verdad que es constitutiva de la teoría tractariana del sentido.

En un planteamiento más sintético (Morris 2008, pp. 44-47) ${ }^{40}$, digamos que, como la proposición "Fa" es una parte integral de la proposición "Es posible que $\mathrm{Fa}$ ", tenemos primero:

(13) La proposición "Es posible que Fa" tiene sentido sólo si la proposición "Fa" tiene sentido.

Además, el consecuente de $\left(\mathrm{A}^{*}\right)$ es equivalente a:

(14) La proposición "Fa" tiene sentido sólo si la proposición "Es posible que $\mathrm{Fa}$ " es verdadera.

Por transitividad de los condicionales (13) y (14), obtenemos finalmente:

(15) La proposición "Es posible que Fa" tiene sentido sólo si la proposición "Es posible que Fa" es verdadera (por 13 y 14).

\footnotetext{
40 A diferencia de Morris, no formularé (13)-(15) como bicondicionales sino como condicionales para subrayar la idea de dependencia de derecha a izquierda que está detrás de cada condicional. Téngase en cuenta, además, que la argumentación de Morris sigue otra línea: para él, la importancia de (15) es que es incompatible con el antecedente de T 2.0211, que según su interpretación es, como vimos, que los objetos no constituyen la forma permanente del mundo (Morris 2008, p. 47). Él no usa (15) sobre la base de que es incompatible con la independencia entre sentido y verdad que es constitutiva de la tesis tractariana del sentido, para de ahí establecer que la proposición "Es posible que Fa" no tendría sentido en primer lugar. Él usa (15) sobre la base de que es incompatible con el antecedente de T 2.0211, para de ahí negar directamente el antecedente.
}

Manuscrito - Rev. Int. Fil. Campinas, v. 41, n. 1, pp. 33-66, jan.-mar. 2018. 
Sin embargo, (15) es incompatible con la independencia entre sentido y verdad que es constitutiva de la tesis tractariana del sentido. En efecto, el carácter esencialmente bipolar de la proposición está en relación con la determinación del sentido independientemente de la verdad (Notes on Logic, pp. 93-94). El sentido es independiente de la verdad en la medida en que una proposición puede tener sentido y ser falsa, puesto que el sentido es independiente de lo que es el caso. Se puede entender una proposición sin saber si es verdadera ( $\mathrm{T}$ 4.024). De ahí que Wittgenstein afirme que una proposición puede ser verdadera $o$ falsa sólo en tanto que ya es una figura de la realidad ( $T$ 4.06). Precisamente porque no se puede reconocer a partir de la figura sola si es verdadera o falsa, no hay figura alguna que sea verdadera a priori (T 2.224-2.225, 6. 113). Para reconocer si la figura es verdadera o falsa tenemos que compararla con la realidad (T 2.223, 4.05). Pues, su verdad o falsedad consiste en el acuerdo o desacuerdo de su sentido con la realidad (T 2.222). De manera que, dado (15), se sigue de la teoría tractariana del sentido que la proposición "Es posible que Fa" no tendría sentido en primer lugar. Tenemos así que si el sentido de la proposición " $\mathrm{Fa}$ " dependiera de la verdad de la proposición "Es posible que Fa", como establece (14), entonces " $F a$ " no podría tener sentido. En efecto, a causa del resultado según el cual su sentido no sería independiente de su propia verdad, la proposición "Es posible que Fa", de cuya verdad dependería el sentido de "Fa", no tendría sentido como para poder ser verdadera (o falsa). Pero habíamos empezado por suponer que una proposición con el nombre "a" (como "Fa") tiene sentido, contrariamente a (B). Por tanto,

(C*) El objeto $a$ es independiente en tanto que puede ocurrir en cualquier situación. Por ello no puede ser compuesto (T 2.021) $\left(\text { por } A^{*} \text { y B }\right)^{41}$.

\footnotetext{
${ }^{41}$ Supongamos por un momento que la proposición "Es posible que Fa" tiene sentido. Entonces, por la independencia entre sentido y verdad que es constitutiva de la teoría tractariana del sentido, deberíamos negar (15). Pero, puesto que la verdad de (13) no está en cuestión, de la negación de (15) se seguiría la negación de (14). Es decir, de la negación de (15) se seguiría la negación del consecuente de $\left(A^{*}\right)$. En consecuencia, por modus tollens se llega a la negación del antecedente de $\left(\mathrm{A}^{*}\right)$. Esta sería, por tanto, otra forma de alcanzar la conclusión $\left(C^{*}\right)$. El problema es que, como ya señalamos, el
} 
Pues, un complejo es un agregado de cosas cuya existencia implica precisamente que se den en la realidad determinadas situaciones o estados de cosas. Por tanto, no es independiente de lo que es el caso.

\section{Referencias}

Almog, J., Perry, J. \& Wettstein, H. (eds.). Themes from Kaplan. Oxford: Oxford University Press, 1989.

Carruthers, P. The Metaphysics of the Tractatus. Cambridge: Cambridge University Press, 1990.

Cerezo, M. The Possibility of Language. Exploring Internal Tensions in Wittgenstein's Tractatus. Stanford: CSLI, 2005.

"Possibility and Logical Space in the Tractatus". International Journal of Philosophical Studies, 20, 5, pp. 645-659, 2012.

Frascolla, P. "On the Nature of Tractatus Objects". Dialectica, 58, 3, pp. 369382, 2004.

GALE, R. M. “Could Logical Space Be Empty?”. En J. Hintikka (ed.) (1976), pp. 85-104.

GINET, C. "An Incoherence in the Tractatus". Canadian Journal of Philosophy, 3, pp. 143-151, 1973.

HintikKA, J. (ed.). Essays on Wittgenstein in Honour of G. H. von Wright. Amsterdam: North-Holland, 1976.

JOHNSTON, C. "Symbols in Wittgenstein's Tractatus". European Journal of Philosophy, 15, 3, pp. 367-394, 2007.

Kaplan, D. "Demonstratives". En J. Almog, J. Perry, H. Wettstein (eds.) (1989), pp. 481-563.

supuesto de que la proposición "Es posible que Fa" tiene sentido parece ser extraña a ciertas ideas centrales del Tractatus.

Manuscrito - Rev. Int. Fil. Campinas, v. 41, n. 1, pp. 33-66, jan.-mar. 2018. 
MAury, A. The concepts of Sinn and Gegenstand in Wittgenstein's Tractatus. Amsterdam: North-Holland, 1977.

Morris, M. Routledge Philosophy Guidebook to Wittgenstein and the Tractatus LogicoPhilosophicus. Londres y Nueva York: Routledge, 2008.

PAGE, J. “Unconfigured Tractarian Objects”. Philosophical Investigations, 20, 1, pp. 39-50, 1997.

ProOPS, I. Logic and Language in Wittgenstein's Tractatus. Nueva York y Londres: Garland Publishing, 2000.

"Wittgenstein on the Substance of the World". European Journal of Philosophy, 12, 1, pp. 106-126, 2004.

REINHARDT, L. “The Impossible Bottom Line”. Analysis, 65, 4, pp. 341-342, 2005.

RICKETTS, T. "Pictures, Logic, and the Limits of Sense in Wittgenstein's Tractatus". En H. Sluga y D. G. Stern (eds.), pp. 59-99.

Sluga, H. \& STERn, D. G. (eds.). The Cambridge Companion to Wittgenstein. Cambridge: Cambridge University Press, 1996.

TEJEDOR, C. "Sense and Simplicity: Wittgenstein's Argument for Simple Objects". Ratio, XVI, 3, pp. 272-289, 2003.

WeDIN, M. V. "Trouble in Paradise? On the Alleged Incoherence of the Tractatus".Grazer Philosophische Studien, 42, pp. 23-55, 1992.

WiTTGENSTEIN, L. Tractatus Logico-Philosophicus. Introducción, traducción y notas de L. M. Valdés Villanueva. 2a edición. Madrid: Tecnos, 2003.

Notebooks 1914-1916. Notes on Logic. G. H. von Wright y G. E. M. Anscombe (eds.). Oxford: Basil Blackwell, 1961.

Wittgenstein's Lectures Cambridge 1930-1932. From the notes of John King and Desmond Lee. D. Lee (ed.). Chicago: University of Chicago Press, 1989.

Zalabardo, J. L. (ed.). Wittgenstein's Early Philosophy. Oxford: Oxford University Press, 2012.

Manuscrito - Rev. Int. Fil. Campinas, v. 41, n. 1, pp. 33-66, jan.-mar. 2018. 
"Reference, Simplicity, and Necessary Existence in the Tractatus". En J. L. Zalabardo (ed.) (2012), pp. 119-150.

Representation and Reality in Wittgenstein's Tractatus. Oxford: Oxford University Press, 2015. 